\title{
Stability Criteria and Turbulence Paradox Problem For Type II 3D Shears
}

\author{
Y. Charles Li
}

\begin{abstract}
There are two types of 3D shears in channel flows: $(U(y, z), 0,0)$ and $(U(y), 0, W(y))$. Both are important in organizing the phase space structures of the channel flows. Stability criteria of the type I 3D shears were studied in [Li, 2010]. Here we study the stability criteria of the type II 3D shears. We also provide more support to the idea of resolution of a turbulence paradox, introduced in [ $\mathrm{Li}$ and Lin, 2010], by studying a sequence of type II 3D shears.
\end{abstract}

\section{Introduction}

Studying the phase space structures of channel flows is an important and emerging area. Most of the existing works in this area is numerical. Like every other dynamical system study in a phase space, non-wandering objects like fixed points, periodic orbits etc. play a fundamental role in organizing the phase space structure. When the Reynolds number is infinite (i.e. zero viscosity), the corresponding 3D Euler equations have two types of steady shears as fixed points in the phase space.

- Type I 3D shears $(U(y, z), 0,0)$,

- Type II 3D shears $(U(y), 0, W(y))$;

where the boundaries of the channel are in the $y$-direction. When the Reynolds number is not infinite but large, these shears turn into slowing drifting states under the corresponding 3D Navier-Stokes dynamics. By the concepts of rate condition and normal hyperbolicity of Fenichel [1 2], these slowing drifting states can be crucial in the transition to turbulence. Also the 3D Navier-Stokes dynamics has fixed points like the so-called lower and upper branches [9]. When the Reynolds number approaches infinity, the lower branch approaches one of the type I 3D shears. How to distinguish this particular one from the rest of the type I 3D shears is an interesting question (also posted in a list of problems by Yudovich [10). A condition satisfied by this particular type I 3D shear was derived in [7. Again like every other dynamical system study in a phase space, the stability of these 3D shears is crucial in understanding the phase space structure. The stability criteria

1991 Mathematics Subject Classification. Primary 76, 37; Secondary 35.

Key words and phrases. Type II 3D shear, turbulence paradox, channel flow, hydrodynamic stability, phase space.

(C) 2010 (copyright holder) 
for type I 3D shears were studied in $\mathbf{5}$. Here we shall study the stability criteria for type II 3D shears. It turns out that the linearized 3D Euler equations can be casted into formally the same form as the classical Rayleigh equation for 2D shears $(U(y), 0)$. But the nature of the stability criteria is fundamentally different from that for the 2D shears. One can ask the question: What is the "percentage" among e.g. all type I 3D shears, that is unstable? The author's conjecture is:

- The unstable percentage of type II 3D shears $>$ the unstable percentage of type I 3D shears $>$ the unstable percentage of $2 \mathrm{D}$ shears.

Finally, we shall provide more support to the idea of resolution of the turbulence paradox, introduced in [6] by studying a sequence of type II 3D shears. The turbulence paradox is also called Sommerfeld paradox which roughly says that the linear shear in the plane Couette flow is linearly stable for all values of the Reynolds number, while in experiments, transition from the linear shear to turbulence occurs when the Reynolds number is large enough. For more details on the turbulence paradox and our resolution, see Section 4 and the paper [6].

\section{Necessary Conditions For Instability}

The inviscid channel flow is governed by the 3D Euler equations

$$
\partial_{t} u_{i}+u_{j} u_{i, j}=-p_{, i}, \quad u_{i, i}=0 ;
$$

where $\left(u_{1}, u_{2}, u_{3}\right)$ are the three components of the fluid velocity along $(x, y, z)$ directions, and $p$ is the pressure. The boundary condition is the so-called nonpenetrating condition

$$
u_{2}(x, a, z)=0, \quad u_{2}(x, b, z)=0 ;
$$

where $a<b$ are the boundary locations of the channel in $y$-direction.

We start with the type II 3D steady shear solutions of the 3D Euler equations:

$$
u_{1}=U(y), \quad u_{2}=0, \quad u_{3}=W(y), \quad p=p_{0} \text { (a constant). }
$$

Of particular importance are those profiles which also satisfy the non-slip boundary condition

$$
U(a)=\alpha, \quad U(b)=\beta, \quad W(a)=W(b)=0 ;
$$

where $\alpha$ and $\beta$ are the velocities of the two walls of the channel. Such profiles may be the viscous limiting profiles when the viscosity approaches zero. Linearize the 3D Euler equations with the notations

$$
\begin{aligned}
& u_{1}=U(y)+\left[e^{i k_{1} x+i k_{3} z-i \sigma t} u(y)+c . c .\right], u_{2}=e^{i k_{1} x+i k_{3} z-i \sigma t} v(y)+c . c . \\
& u_{3}=W(y)+\left[e^{i k_{1} x+i k_{3} z-i \sigma t} w(y)+c . c .\right], p \rightarrow p_{0}+\left[e^{i k_{1} x+i k_{3} z-i \sigma t} p(y)+c . c .\right] ;
\end{aligned}
$$

where $k_{1}$ and $k_{3}$ are the wave numbers, and $\sigma$ is a complex constant; we obtain the linearized 3D Euler equations

$$
\begin{aligned}
& i\left(k_{1} U+k_{3} W-\sigma\right) u+U^{\prime} v=-i k_{1} p, \\
& i\left(k_{1} U+k_{3} W-\sigma\right) v=-p^{\prime}, \\
& i\left(k_{1} U+k_{3} W-\sigma\right) w+W^{\prime} v=-i k_{3} p, \\
& i k_{1} u+v^{\prime}+i k_{3} w=0 .
\end{aligned}
$$

Two forms of simplified systems can be derived:

$$
v^{\prime \prime}-\frac{k_{1} U^{\prime \prime}+k_{3} W^{\prime \prime}}{k_{1} U+k_{3} W-\sigma} v=\left(k_{1}^{2}+k_{3}^{2}\right) v,
$$


with the boundary condition $v(a)=v(b)=0$; and

$$
\left(k_{1} U+k_{3} W-\sigma\right)^{2}\left[\left(k_{1} U+k_{3} W-\sigma\right)^{-2} p^{\prime}\right]^{\prime}=\left(k_{1}^{2}+k_{3}^{2}\right) p,
$$

with the boundary condition $p^{\prime}(a)=p^{\prime}(b)=0$.

Notice that formally equation (2.7) is in the same form as the clasical Rayleigh equation where the $2 \mathrm{D}$ shear $U(y)$ is now replaced by $k_{1} U+k_{3} W$. We have the following extension of the classical Rayleigh's inflection-point theorem.

Theorem 2.1. If $\left(k_{1}, k_{3}\right)$ is an unstable mode, then

$$
\left(k_{1} U^{\prime \prime}+k_{3} W^{\prime \prime}\right)\left(y_{*}\right)=0 \text { for some } y_{*} \in(a, b) \text {. }
$$

Remark 2.2. Even though the Rayleigh's inflection-point theorem is a special case of the above theorem, the claim in the above theorem in general is fundamentally different from that of Rayleigh. Rayleigh's claim basically says that if $(U(y), 0)$ is linearly unstable, then $U(y)$ has an inflection point. Here on the other hand, condition (2.9) can be satisfied by most of type II 3D shears $(U(y), 0, W(y))$. In fact, one can choose $k_{1}=W^{\prime \prime}\left(y_{*}\right), k_{3}=-U^{\prime \prime}\left(y_{*}\right)$ for any $y_{*} \in(a, b)$, then (2.9) is satisfied. Therefore, in general (2.9) is a very weak necessary condition for the linear instability of $(U(y), 0, W(y))$. This may indicate that type II $3 \mathrm{D}$ shears are more often to be unstable than $2 \mathrm{D}$ shears. The author's conjecture is that type I $3 \mathrm{D}$ shears are inbetween in terms of frequency of instability.

Proof. Multiplying (2.7) by $\bar{v}$,

$$
\int_{a}^{b}\left[\left|v^{\prime}\right|^{2}+\left(k_{1}^{2}+k_{3}^{2}\right)|v|^{2}\right] d y+\int_{a}^{b} \frac{k_{1} U^{\prime \prime}+k_{3} W^{\prime \prime}}{k_{1} U+k_{3} W-\sigma}|v|^{2} d y=0
$$

the imaginary part of which is

$$
\sigma_{i} \int_{a}^{b} \frac{k_{1} U^{\prime \prime}+k_{3} W^{\prime \prime}}{\left|k_{1} U+k_{3} W-\sigma\right|^{2}}|v|^{2} d y=0,
$$

where $\sigma=\sigma_{r}+i \sigma_{i}$. Thus

$$
\left(k_{1} U^{\prime \prime}+k_{3} W^{\prime \prime}\right)\left(y_{*}\right)=0 \text { for some } y_{*} \in(a, b) .
$$

We also have the following extension of the Fjortoft's theorem.

Theorem 2.3. If $\left(k_{1}, k_{3}\right)$ is an unstable mode, then

$$
G^{\prime \prime}\left(y_{0}\right)\left[G\left(y_{0}\right)-G\left(y_{*}\right)\right]<0 \quad \text { for some } y_{0} \in(a, b),
$$

where $G=k_{1} U+k_{3} W$ and $y_{*}$ is the point at which $G^{\prime \prime}=0$ given by Theorem 2.1.

Proof. The real part of equation (2.10) is

$$
\int_{a}^{b} \frac{G^{\prime \prime}\left(G-\sigma_{r}\right)}{|G-\sigma|^{2}}|v|^{2} d y=-\int_{a}^{b}\left[\left|v^{\prime}\right|^{2}+\left(k_{1}^{2}+k_{3}^{2}\right)|v|^{2}\right] d y .
$$

From (2.11), one has

$$
\left[\sigma_{r}-G\left(y_{*}\right)\right] \int_{a}^{b} \frac{G^{\prime \prime}}{|G-\sigma|^{2}}|v|^{2} d y=0
$$


where $y_{*}$ is chosen to be the point given by Theorem 2.1 $G^{\prime \prime}\left(y_{*}\right)=0$. The above two equations imply that

$$
\int_{a}^{b} \frac{G^{\prime \prime}(y)\left[G(y)-G\left(y_{*}\right)\right]}{|G(y)-\sigma|^{2}}|v|^{2} d y<0
$$

and the theorem is proved.

Next we show the extension of Howard's semi-circle theorem.

Theorem 2.4. If $\left(k_{1}, k_{3}\right)$ is an unstable mode, then its corresponding unstable eigenvalue lies inside the semi-circle in the complex plane:

$$
\left(\sigma_{r}-\frac{M+m}{2}\right)^{2}+\sigma_{i}^{2} \leq\left(\frac{M-m}{2}\right)^{2}
$$

where again $\sigma=\sigma_{r}+i \sigma_{i}, M=\max _{y \in[a, b]}\left(k_{1} U+k_{3} W\right)$, and $m=\min _{y \in[a, b]}\left(k_{1} U+\right.$ $\left.k_{3} W\right)$.

Proof. Multiply (2.8) with $\bar{p}(G-\sigma)^{-2}$, integrate by parts, and split into real and imaginary parts; we obtain that

$$
\begin{aligned}
& \int_{a}^{b} G Q d y=\sigma_{r} \int_{a}^{b} Q d y \\
& \int_{a}^{b} G^{2} Q d y=2 \sigma_{r} \int_{a}^{b} G Q d y+\left(\sigma_{i}^{2}-\sigma_{r}^{2}\right) \int_{a}^{b} Q d y \\
& =\left(\sigma_{r}^{2}+\sigma_{i}^{2}\right) \int_{a}^{b} Q d y
\end{aligned}
$$

by (2.12), where

$$
\begin{aligned}
G & =k_{1} U+k_{3} W, \\
Q & =|G-\sigma|^{-4}\left[\left|p^{\prime}\right|^{2}+\left(k_{1}^{2}+k_{3}^{2}\right)|p|^{2}\right] .
\end{aligned}
$$

Let

$$
M=\max _{y \in[a, b]} G, \quad m=\min _{y \in[a, b]} G,
$$

then

$$
\int_{a}^{b}(G-m)(M-G) Q d y \geq 0 .
$$

Expand this inequality and utilize (2.12)-(2.13), we arrive at the semi-circle inequality in the theorem.

\section{Sufficient Conditions For Instability}

The zeroth mode $\left(k_{1}, k_{3}\right)=(0,0)$ is trivially neutrally stable, so our interest is focused upon non-zero modes. Without loss of generality, we assume $k_{1} \neq 0$. Equation (2.7) can be re-written in the form

$$
v^{\prime \prime}-\frac{U^{\prime \prime}+\frac{k_{3}}{k_{1}} W^{\prime \prime}}{U+\frac{k_{3}}{k_{1}} W-\frac{\sigma}{k_{1}}} v=k_{1}^{2}\left[1+\left(\frac{k_{3}}{k_{1}}\right)^{2}\right] v .
$$

We will keep $\kappa=k_{3} / k_{1}$ fixed and vary $k_{1}$. Denote by

$$
H=U+\kappa W, c=\frac{\sigma}{k_{1}}, \alpha=k_{1} \sqrt{1+\kappa^{2}},
$$


we obtain the following equivalent form of (2.7),

$$
v^{\prime \prime}-\frac{H^{\prime \prime}}{H-c} v=\alpha^{2} v,
$$

with the boundary condition $v(a)=v(b)=0$, which is in the same form as the classical Rayleigh equation [6. Thus we have the extension of the Tollmien's theorem on a sufficient condition for instability.

Theorem 3.1. If $H^{\prime}(y) \neq 0$ for all $y \in(a, b), H^{\prime \prime}\left(y_{*}\right)=0$ for some $y_{*} \in(a, b)$, and the Sturm-Liouville operator

$$
L v=-v^{\prime \prime}+\frac{H^{\prime \prime}}{H-H\left(y_{*}\right)} v
$$

has a negative eigenvalue under the Dirichlet boundary condition $v(a)=v(b)=0$; then the equation (3.1) has an unstable eigenvalue (in fact, an unstable curve $c=$ $c(\alpha)$ for $\alpha$ in some interval).

\section{Turbulence Paradox}

Turbulence paradox is also called Sommerfeld paradox. It originated from Sommerfeld's analysis which concluded that the linear shear in plane Couette flow is linearly stable for all values of the Reynolds number; whereas in fluid experiments, perturbations of the linear shear often lead to transition to turbulence. Such a paradox is universal among fluid flows, e.g. pipe Poiseuille flow, plane Poiseuille flow etc.. A resolution of this paradox is given in $\mathbf{6}$. The main idea of the resolution is to show that even though the linear shear is linearly stable, states arbitrarily close to the linear shear can still be linearly unstable. Here different norms are crucial. It is shown in [6] that the sequence of $2 \mathrm{D}$ shears $\left(U_{n}(y), 0\right)$ is linearly unstable for all $n$ and large enough Reynolds number (including infinity), where

$$
U_{n}(y)=y+\frac{A}{n} \sin (4 n \pi y),\left(\frac{1}{2} \frac{1}{4 \pi}<A<\frac{1}{4 \pi}\right) .
$$

Here $U_{n}(y)$ approaches the linear shear $U=y$ in the $L^{\infty}$ norm of velocity, but not in the $L^{\infty}$ norm of vorticity. Notice also that when the Reynolds number is not infinite but large, the shears $\left(U_{n}(y), 0\right)$ are not steady states rather slowly drifting states. The linear instability mentioned above is predicted by the Orr-Sommerfeld operator (linearized 2D Navier-Stokes operator) when the shears $\left(U_{n}(y), 0\right)$ are viewed frozen. Such an instability is in the spirit of Fenichel's rate condition and normal hyperbolicity [1] [2]. Such an instability is also observed numerically [4].

In this paper, we would like to add more support to the idea of resolution mentioned above by considering the type II 3D shears $(U(y), 0, W(y))$.

The viscous channel flow is governed by the Navier-Stokes equations

$$
\partial_{t} u_{i}+u_{j} u_{i, j}=-p_{, i}+\epsilon u_{i, j j}, \quad u_{i, i}=0 ;
$$

where again $\left(u_{1}, u_{2}, u_{3}\right)$ are the three components of the fluid velocity along $(x, y, z)$ directions, $p$ is the pressure, and $\epsilon=1 / R$ is the inverse of the Reynolds number $R$. The boundary condition is

$$
u_{1}(x, a, z)=\alpha, \quad u_{1}(x, b, z)=\beta, \quad u_{j}(x, a, z)=u_{j}(x, b, z)=0,(j=2,3) ;
$$


where $a<b, \alpha<\beta$. For the viscous channel flow, the type II 3D shears mentioned above are no longer fixed points, instead they drift slowly in time (sometimes called quasi-steady solutions):

$$
\left(e^{\epsilon t \partial_{y}^{2}} U(y), 0, e^{\epsilon t \partial_{y}^{2}} W(y)\right) .
$$

By ignoring the slow drift and pretending they are still fixed points (or by using artificial body forces to stop the drifting), their unstable eigenvalues will lead to transient nonlinear growths as shown numerically [4]. A better explanation here is to use the theory of geometric singular perturbation [1 $[2,3,8]$. The slowly drifting 3D shears altogether form a locally invariant slow (center) manifold. The normal direction growth rate (or decay rate) of this slow manifold has a persistence property (i.e. robust). Thus the growth rate can be estimated by ignoring the slow drift. The geometric singular perturbation theory implies the transient nonlinear growth induced by the linear growth rate.

The corresponding linear Navier-Stokes operator at $(U(y), 0, W(y))$ is given by the following counterpart of (2.3)-(2.6),

$$
\begin{aligned}
& i\left(k_{1} U+k_{3} W-\sigma\right) u+U^{\prime} v=-i k_{1} p+\epsilon\left[u^{\prime \prime}-\left(k_{1}^{2}+k_{3}^{2}\right) u\right], \\
& i\left(k_{1} U+k_{3} W-\sigma\right) v=-p^{\prime}+\epsilon\left[v^{\prime \prime}-\left(k_{1}^{2}+k_{3}^{2}\right) v\right], \\
& i\left(k_{1} U+k_{3} W-\sigma\right) w+W^{\prime} v=-i k_{3} p+\epsilon\left[w^{\prime \prime}-\left(k_{1}^{2}+k_{3}^{2}\right) w\right], \\
& i k_{1} u+v^{\prime}+i k_{3} w=0 .
\end{aligned}
$$

The simplified system as the counterpart of (2.7) is

$$
\frac{\hat{\epsilon}}{i \alpha}\left[\frac{d^{2}}{d y^{2}}-\alpha^{2}\right]^{2} v+H^{\prime \prime} v-(H-c)\left[\frac{d^{2}}{d y^{2}}-\alpha^{2}\right] v=0,
$$

with the boundary condition $v=v^{\prime}=0$ at $y=a, b$; where $\hat{\epsilon}=\epsilon \sqrt{1+\kappa^{2}}$, as before $\kappa=k_{3} / k_{1}$ (fixed), $\alpha=k_{1} \sqrt{1+\kappa^{2}}, c=\sigma / k_{1}$, and $H=U+\kappa W$. Now both equation (3.1) and equation (4.8) are formally in the same form as those in [6]. We can specify

$$
a=0, b=1, \alpha=0, \beta=1 ;
$$

and consider the following sequence of type II $3 \mathrm{D}$ shears

$$
\left(U_{n}(y), 0, W_{n}(y)\right)=\left(y, 0, \frac{A}{n} \sin (4 n \pi y)\right) .
$$

The problem of linear instability of the type II 3D shears (4.9) is then casted into the same problem as that of the $2 \mathrm{D}$ shears (4.1). By the results of [6], we have

Theorem 4.1. For any $A>0$ and any integer $n \geq 1$, the type II $3 D$ shears (4.9) are linearly unstable under the $3 D$ Euler dynamics. Specifically, there exists a $2 D$ unstable eigenmode surface $\left(k_{1}, k_{3} ; \sigma\left(k_{1}, k_{3}\right)\right)$ with $\operatorname{Im}\left\{\sigma\left(k_{1}, k_{3}\right)\right\}>0$ for the equation (2.7), stemming from neutral modes of the form $\left(k_{1}(n), k_{3}(n) ; \frac{1}{2} k_{1}(n)\right)$ where $\left[k_{1}(n)\right]^{2}+\left[k_{3}(n)\right]^{2} \geq C n^{2}(C>0$ is independent of $n)$. The corresponding eigenfunctions are in $C^{\infty}(0,1)$.

Proof. Fix $\kappa=k_{3} / k_{1}$ such that

$$
\frac{1}{2} \frac{1}{4 \pi}<\kappa A<\frac{1}{4 \pi},
$$

then the problem is reduced to that of Theorem 3.2 in [6]. 
Theorem 4.2. Let $\left(k_{1}^{0}, k_{3}^{0} ; \sigma^{0}\right)$ be a point on the $2 D$ unstable eigenmode surface given by Theorem 4.1. Then when $\epsilon$ is sufficiently small, there exists an unstable eigenmode $\left(k_{1}^{0}, k_{3}^{0} ; \sigma^{*}\right)$ with $\operatorname{Im}\left\{\sigma^{*}\right\}>0$ for the equation 4.8$)$ with $H$ given by (4.9). When $\epsilon \rightarrow 0^{+}, \sigma^{*} \rightarrow \sigma^{0}$.

Proof. For the fixed $\left(k_{1}^{0}, k_{3}^{0}\right), \kappa$ has a fixed value; then $\hat{\epsilon}$ and $\epsilon$ are equivalent. The problem is reduced to that of Theorem 4.1 in [6].

Remark 4.3. Notice that the sequence of type II 3D shears (4.9) are linearly unstable for all $A>0$, while the sequence of $2 \mathrm{D}$ shears (4.1) are proved linearly unstable for $\frac{1}{2} \frac{1}{4 \pi}<A<\frac{1}{4 \pi}$. The sequence of type II 3D shears (4.9) also approaches the linear shear $U=y$ in the $L^{\infty}$ norm of velocity, but not in the $L^{\infty}$ norm of vorticity.

\section{References}

[1] N. Fenichel, Asymptotic stability with rate conditions, Ind. Univ. Math. J. 23 (1974), 11091137.

[2] N. Fenichel, Asymptotic stability with rate conditions II, Ind. Univ. Math. J. 26 (1977), 81-93.

[3] N. Fenichel, Geometric singular perturbation theory for ordinary differential equations, $J$. Diff. Eq. 31 (1979), 53-98.

[4] Y. Lan, Y. Li, Sommerfeld paradox - a novel numerical study, in progress (2010). Available partially at: http://www.math.missouri.edu/ ${ }^{\sim}$ cli/Somnum.pdf

[5] Y. Li, Stability criteria of 3D inviscid shears, in press (2010). Available at: http://www.math.missouri.edu/ cli/S3DS.pdf

[6] Y. Li, Z. Lin, A resolution of the Sommerfeld paradox, Submitted (2010). Available at: http://www.math.missouri.edu/ cli/Som.pdf

[7] Y. Li, D. Viswanath, Exact and asymptotic conditions on traveling wave solutions of the Navier-Stokes equations, Physics of Fluids 21 (2009), 101703.

[8] Y. Li, S. Wiggins, Invariant Manifolds and Fibrations for Perturbed Nonlinear Schrödinger Equations, Springer-Verlag, Applied Mathematical Sciences, vol.128, (1997).

[9] M. Nagata, Three-dimensional finite-amplitude solutions in plane Couette flow: bifurcation from infinity, J. Fluid Mech. 217 (1990), 519-527.

[10] V. Yudovich, Eleven great problems of mathematical hydrodynamics, Moscow Math. J. 3, no.2 (2003), 711-737, problem 11b.

Department of Mathematics, University of Missouri, Columbia, MO 65211, UsA

E-mail address: liyan@missouri.edu 